\title{
Terminological Logic Involving Time and Evolution: A Preliminary Report
}

\author{
Patrick Lambrix and Ralf Rönnquist
}

\section{Linköping University Post Print}

\section{Tweet}

N.B.: When citing this work, cite the original article.

Original Publication:

Patrick Lambrix and Ralf Rönnquist, Terminological Logic Involving Time and Evolution: A Preliminary Report, 1993, in Methodologies for Intelligent Systems, 162-171.

http://dx.doi.org/10.1007/3-540-56804-2_16

Copyright: Springer

Postprint available at: Linköping University Electronic Press

http://urn.kb.se/resolve?urn=urn:nbn:se:liu:diva-119312 


\title{
Terminological Logic Involving Time and Evolution : A Preliminary Report
}

\author{
Patrick Lambrix and Ralph Rönnquist \\ Department of Computer Science \\ Linköping University \\ S-581 83 Linköping \\ SWEDEN
}

email: patla @ ida.liu.se, ralro @ ida.liu.se

\begin{abstract}
Although terminological logics as well as temporal reasoning has received considerable attention in the knowledge representation community in the last years, few attempts have been made to integrate those fields. We study the combination of the temporal logic LITE and a terminological logic to obtain a temporal terminological logic.

We emphasize defining a terminological logic (T-LITE) where the extensions of concepts are time-dependent in the following sense : first, the individuals belonging to a concept are appearances of objects in a temporal context; secondly, we allow concepts to be defined in terms of developments of objects. Formal semantics for T-LITE are provided.
\end{abstract}

This is a preprint of the paper published by Springer:

Lambrix P, Rönnquist R, Terminological Logic Involving Time and Evolution: A Preliminary Report, Proceedings of the Seventh International Symposium on Methodologies for Intelligent Systems ISMIS93, LNAI 689, 162-171, Trondheim, Norway, 1993. The final publication is available at www.springerlink.com.

doi: 10.1007/3-540-56804-2_16 


\section{Introduction}

Although terminological logics as well as temporal reasoning has received considerable attention in the knowledge representation community in the last years, few attempts have been made to integrate those fields. We study the combination of LITE (Logic Involving Time and Evolution) semantics and a base terminological logic so as to extend terminological logic with concepts regarding time and evolution.

Terminological logics or concept languages are languages tailored for expressing knowledge about concepts and concept hierarchies. They are usually given a Tarski style declarative semantics (see eg. [Neb90, App A]), which allows them to be seen as sub-languages of predicate logic. One starts with primitive concepts and roles, and can use the language constructs (such as intersection, union, role quantification etc.) to define new concepts and roles. Concepts can be considered as unary predicates which are interpreted as sets of individuals whereas roles are binary predicates which are interpreted as binary relations between individuals. The basic reasoning tasks are unsatisfiability and subsumption checking. A concept is unsatisfiable if it always denotes an empty set. A concept $C$ is subsumed by a concept $D$ if the extension of $C$ is always a subset of the extension of $D$.

A whole family of knowledge representation systems have been built using these languages and for most of them complexity results for the subsumption algorithm are known (e.g. KL-ONE[BrS85], BACK [Neb90], CLASSIC [Bo*89], KANDOR [PaS84], KRYPTON [Br*85], LOOM [MGB87]).

'LITE' ([Rön90],[Rön92]) on the other hand, is a variation to first-order predicate logic where in particular the notion of object is revised from being an indivisible entity into being a temporal structure of versions. Each object version is then indivisible and unchanged in time, while an object changes in time by taking different appearances.

When approaching the task of mixing terminological logic with time, we observe that the idea of "temporal concept" requires some analysis. On the one hand, a concept may be time-dependent in its extension, i.e. the set of objects satisfying its definition is different at different times. This is the case usually dealt with (e.g. [Sch90]), and it seems to be in some way a "first" or more immediate case.

A concept may also be time-dependent in its definition, whereby we mean that an object is included in or excluded from the concept extension depending on which development the object shows. For instance, the concept 'traffic-light' defined as a light cycling over being ${ }^{1}$ green, yellow, and red is such a concept.

\footnotetext{
${ }^{1}$ Subject to national variation.
} 
The latter kind of concepts seems to potentially extend beyond the idea of concept languages, because the extension of a concept no longer is a set of single objects but more like a set of history fragments involving several objects in some certain development combinations. For instance, the concept of 'chessgame' denotes scenarios involving (at least) two players, a chess board, and chess pieces, where the latter move over the chess board admitting to a small set of specific rules. To cover these kinds of concepts in terms of terminological logic, one has to reify; to introduce abstract objects that denote scenarios.

Finally, a concept may also change in itself, so that its extension changes because its definition changes. For instance the concept of (legally) 'adult' may change by decreasing the required age. This kind of development is probably even more difficult to deal with within terminological logic, since for one thing, it means that subsumption relationships change.

In our study here we use the same base concept language as [Sch90], which formally is a subset of most current terminological logics. However while the focus in [Sch90] is on the ability to express extensional concept changes by using explicit time references, we focus on using more implicit temporal qualifications for object selection in the sense of classification ${ }^{2}$. Further we make a start in tackling the second kind of time-dependent concepts, i.e. where an object is included in or excluded from the concept extension depending on which development the object shows. In section 2 we describe the temporal logic LITE. Then we use the LITE semantics to define in section 3 the temporal terminological logic T-LITE. We conclude the paper with an example (section 4) and a discussion (section 5).

\section{LITE}

The LITE ([Rön92]) logic is a first-order predicate logic with an extension allowing objects to be seen as sets of versions. The syntax of the temporal framework is the normal syntax of first-order predicate logic extended by the use of the temporal operator @. In principle, the normal first order logic semantics is also used, but over versions, rather than over objects.

Formally, a structure is a tuple (Vs,Obj,Col,Rel,Func). Vs is the set of distinct individuals that correspond to the appearances or versions of the objects. We have a partial time order between versions. Versions belonging to the same object which are not ordered by the partial time order are said to be parallel versions. Obj is the set of objects. An object is a set of versions. All objects are pairwise disjoint. Col is the set of collections. A collection is a set composed of exactly one version of each object such that all those versions are potentially

\footnotetext{
${ }^{2}$ It may of course be useful in practice to allow clock metrics, but in our approach, such metric is contained within the data rather than extending the representation language.
} 
contemporary (see below). The version of object $\mathrm{x}$ in the collection $\mathrm{t}$ is denoted by x@t. A collection can be seen as a possible time-slice over the objects. Func is the set of functions. A function has its arguments in Vs or Col and its image in Obj. Rel is the set of relations. A relation has its arguments in Vs or Col.

A formula is interpreted with respect to a structure $S$, a collection $t$ in the structure and a binding $h$ which maps variables to objects and time variables to collections. The principle is to interpret formulas in the classical way with the exception that object references are disambiguated to versions by intersecting the object with the collection $t$.

Figure 1: ordering between collections

By revising the notion of object from being an indivisible entity into being a temporal structure of versions, mixed time relations are easily expressed in LITE. A sentence like "Now, I like young Plato but I don't like old Plato" can be expressed in LITE as follows.

$\forall \mathrm{t}:(($ Young(Plato@t) $\rightarrow$ Like(me@now,Plato@t) $)$

$\wedge($ Old(Plato@t $) \rightarrow \neg$ Like(me@now,Plato@t $)))$

We see that in this sentence three different tenses are at work, indicated by 'now', 'young' and 'old'. With temporal indexing on objects, 'now' qualifies 'I' while 'young' and 'old' are different qualifications for Plato.

Due to the importance of the 'collection' notion, we give the formal definition [Rön92].

\section{Definition}

A set $\mathrm{u}$ of one version of each object is a collection iff there is no object $\mathrm{x}$ whose version $\mathrm{x} @ \mathrm{u}$ in $\mathrm{u}$ is succeeded by a version $\alpha$ of $\mathrm{x}$ that precedes the version $y @ u$ of another object $y$ in $u$. 
The time ordering of versions induces an ordering on the collections such that $\mathrm{t} 1 \preceq \mathrm{t} 2$ for collections $\mathrm{t} 1$ and $\mathrm{t} 2$ if for all objects $\mathrm{x}: \mathrm{x} @ \mathrm{t} 1 \preceq \mathrm{x} @ \mathrm{t} 2$. We note that not all collections are related to each other in this way. If $\mathrm{t} 1 \npreceq \mathrm{t} 2$ and $\mathrm{t} 2 \npreceq \mathrm{t} 1$ then $\mathrm{t} 1$ and $\mathrm{t} 2$ are regarded as parallel collections. This could for instance represent different possible ways in which the world can have evolved.

Let us assume for the example in figure 1 that $\mathrm{t} 1=\{\mathrm{x} 1, \mathrm{y} 1\}, \mathrm{t} 2=\{\mathrm{x} 2, \mathrm{y} 2\}$ and $\mathrm{t} 3=\{\mathrm{x} 3, \mathrm{y} 2\}$. Then we have $\mathrm{t} 1 \preceq \mathrm{t} 2, \mathrm{t} 1 \preceq \mathrm{t} 3$, and $\mathrm{t} 2$ and $\mathrm{t} 3$ are parallel collections. An interval $[\mathrm{t} 1, \mathrm{t} 2]$ for $\mathrm{t} 1 \preceq \mathrm{t} 2$ is the set of collections $\mathrm{t}$ such that $\mathrm{t} 1 \preceq \mathrm{t} \preceq \mathrm{t} 2$.

In the rest of this paper we sometimes write "an object at time t" where we actually mean "the version of the object in collection t".

\section{A Temporal Terminological Logic}

In our attempt to combine the LITE semantics with terminological logic, we define a term valuation function relative to a structure so that the value of a concept is a set of pairs $\langle d, t\rangle$ of objects $d$ and collections $t$. We call such a pair a pointer which points to the version $d @ t$.

\section{Table 1}
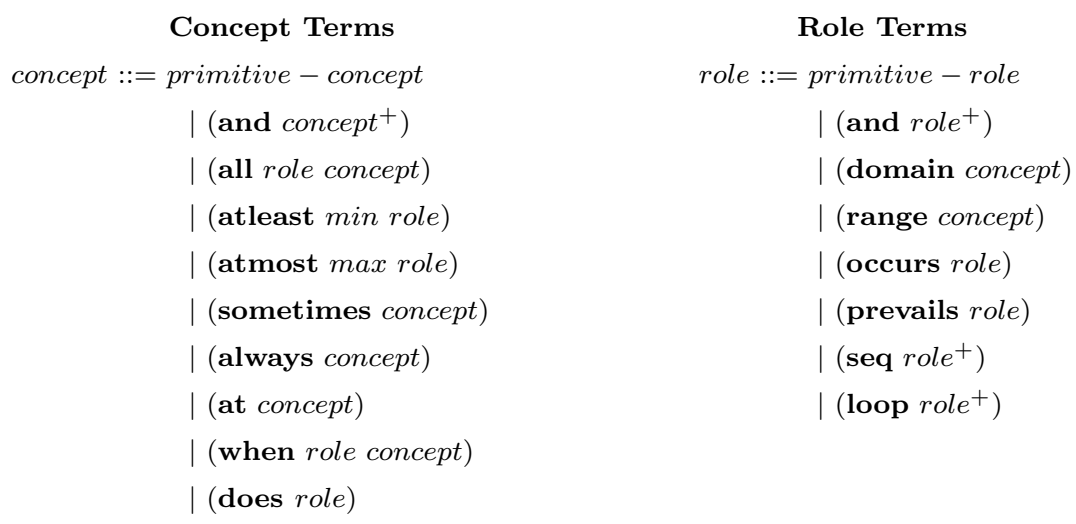

A concept term is a term whose value is a set of pointers; namely the pointers satisfying the particular selection conditions of the term. A role term is a term whose value is a set of pointer pairs such that the pairs satisfy the role term definition. Our language accepts the concept and role terms shown in table 1. Their formal semantics are defined as in table 2 .

Concepts and roles may also be primitive ${ }^{3}$, in which case their values are

\footnotetext{
${ }^{3}$ In [Neb90] a primitive concept is a concept for which the meaning of the concept can only be partially determined by its description. It seems to serve the same purpose in the sense that all concepts and roles are defined directly or indirectly from the primitive concepts and roles.
} 
directly retrievable in the structure; the concepts as sets of pointers and the roles as sets of pairs of pointers. Intuitively a primitive concept is seen as a property of an object version, and a primitive role is seen as a binary relation between object versions.

\section{Table 2}

\section{Standard Terms}

$D$ is the domain set of all pointers.

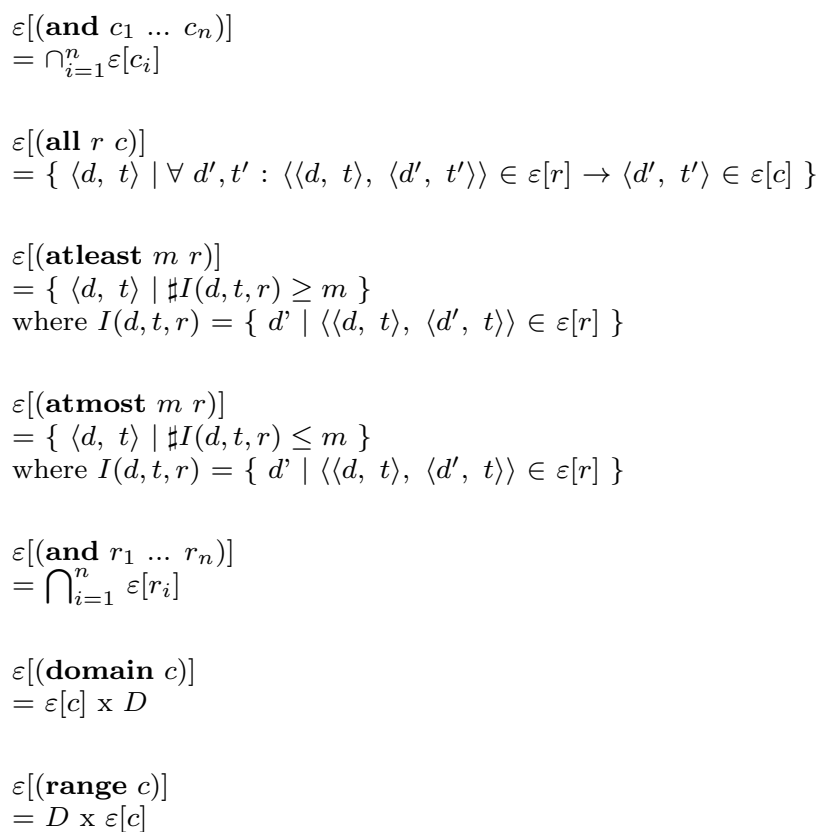

$\varepsilon[$ (sometimes $c)]$

$=\left\{\langle d, t\rangle \mid \exists t^{\prime}:\left\langle d, t^{\prime}\right\rangle \in \varepsilon[c]\right\}$

$\varepsilon[$ (always $c)]$

$=\left\{\langle d, t\rangle \mid \forall t^{\prime}:\left\langle d, t^{\prime}\right\rangle \in \varepsilon[c]\right\}$

$\varepsilon[($ at $c)]$

$=\left\{\langle d, t\rangle \mid \exists d^{\prime}:\left\langle d^{\prime}, t\right\rangle \in \varepsilon[c]\right\}$

$\varepsilon[($ when $r c)]$

$=\left\{\langle d, t\rangle \mid \forall d^{\prime}:\left\langle\langle d, t\rangle,\left\langle d^{\prime}, t\right\rangle\right\rangle \in \varepsilon[r] \rightarrow\left\langle d^{\prime}, t\right\rangle \in \varepsilon[c]\right\}$

$\varepsilon[($ occurs $r)]$

$=\left\{\left\langle\langle d, t\rangle,\left\langle d^{\prime}, t\right\rangle\right\rangle \mid \exists t^{\prime}: d @ t=d @ t^{\prime} \wedge\left\langle\left\langle d, t^{\prime}\right\rangle,\left\langle d^{\prime}, t^{\prime}\right\rangle\right\rangle \in \varepsilon[r]\right\}$

$\varepsilon[($ prevails $r)]$

$=\left\{\left\langle\langle d, t\rangle,\left\langle d^{\prime}, t\right\rangle\right\rangle \mid \forall t^{\prime}: d @ t=d @ t^{\prime} \rightarrow\left\langle\left\langle d, t^{\prime}\right\rangle,\left\langle d^{\prime}, t^{\prime}\right\rangle\right\rangle \in \varepsilon[r]\right\}$ 


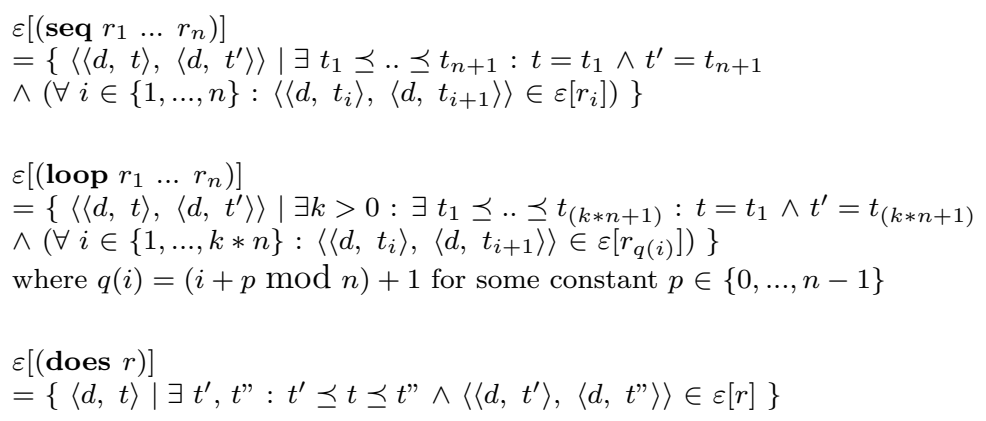

The constructs and, all, atleast, and atmost for concepts and and, domain, and range for roles are regarded as standard terms and we aim for definitions which as much as possible follow the standard definitions for these terms. We do introduce however some amount of temporal dependency; e.g. the counting of role fillers implied by atmost and atleast occurs within collections.

Although these standard terms do not seem to be time dependent in the mnemonics of the operators, they really are of course. Consider for example the following term.

(and male (atleast 1 (and child (range female))))

This term selects the males in versions at the times at which they have atleast 1 daughter. By associating a concept with a set of pointers, we allow a concept to select versions of objects within a temporal context.

The constructs sometimes, always, at, and when for concepts and occurs and prevails for roles are seen as primary temporal terms. They are used for temporal qualifications based on inter object synchronization.

The constructs (sometimes $c$ ) and (always $c$ ) are object selectors, i.e. they select all pointers for a particular object if the object belongs to the concept $c$ at some time, or at all times respectively.

The (at $c$ ) construct is the temporal ${ }^{4}$ counterpart of (sometimes $c$ ). It selects a whole collection whenever some object in that collection satisfies $c$. The at term is for example most useful in connection with more explicit collection references, so that e.g. August-1990 would be a qualification for some collections. This is accomplished by assuming a number of time counter objects. The qualification August may then be used in reference to some specific month counter versions and 1990 to some year counter versions. The concept August-1990 would then be defined as

\footnotetext{
${ }^{4}$ In the sense that it selects collections instead of object.
} 
(and (at August) (at 1990))

Figure 2:

The concept term (when $r c$ ) is in principle the same as (all $r c$ ) but with the addition of the constraint that the role filling pointer is synchronized with the collection in which the role is filled. The difference can easily be seen in the following example. Consider an object with the name PETER. Assume then that a particular version of PETER owns a red car that changes to green ${ }^{5}$ and he owns no other cars (see figure 2). Then the terms

(all owned-car red)

(all owned-car green)

do not select this particular PETER version. On the other hand

(when owned-car red)
(when owned-car green)

select both this particular PETER version although within different temporal context ( $\langle P E T E R, t 1\rangle$ for (when owned-car red) and $\langle P E T E R, t 2\rangle$ for (when owned-car green)).

Whereas sometimes and always are object selectors with respect to concepts, occurs and prevails are version selectors with respect to roles. They

\footnotetext{
${ }^{5}$ This means that we assume that PETER's appearance does not change when he sees his car changing color.
} 
Figure 3:

select all pointers for a particular version as first argument if the version is first argument for the role $r$ at some time, or at all times respectively. The role term occurs extends a contemporary role filling to a contemporary role filling over a whole version. The role term prevails selects pairs only if the role holds for the whole version of the pointer which is first argument.

For the example in figure 2 both

$$
\begin{aligned}
& \text { (prevails owned-car) } \\
& \text { (occurs owned-car) }
\end{aligned}
$$

select $\langle\langle P E T E R, t 1\rangle,\langle C A R, t 1\rangle\rangle$ and $\langle\langle P E T E R, t 2\rangle,\langle C A R, t 2\rangle\rangle$.

For the example in figure 3, (prevails owned-car) does not select any pointer pair. On the other hand (occurs owned-car) extends the role to the whole version and selects $\langle\langle P E T E R, t 1\rangle,\langle C A R, t 1\rangle\rangle$ as well as $\langle\langle P E T E R, t 2\rangle,\langle C A R, t 2\rangle\rangle$.

Finally the constructs does for concepts and seq and loop for roles are introduced as secondary temporal terms allowing for temporal qualification based on intra object synchronization, i.e. object development. These operators might also be called plan forming operators.

The concept term does transforms a succession role into a selection of all the pointers over which the development appears. The outcome of a seq term is the transition from the first to the last object versions between which the indicated development appears. An object changing color from green to yellow to red in succession could for instance be defined by the following term :

(seq (domain green) (domain yellow) (domain red))

Informally, the loop construct recognizes a recurrent development corresponding to a succession of equal sequences. 


\section{Example}

By this example we illustrate two specific issues in using T-LITE. One thing is the ability to recognize and classify objects by means of their development in time. The other thing is the ability to recognize and classify a compound scenario by means of how the developments of components are synchronized. Both of these are issues that emphasize the temporal aspects in a scenario classification.

An intuitively simple kind of object recognized and classified through its development is a traffic light. Informally, a traffic light is a light which continuously changes color from green to yellow to red to green. This can be formulated in T-LITE as follows. (We use ::= as a definition symbol.)

traffic-light $::=$

(and light

(always

(does

(loop

(domain green)

(domain yellow)

$($ domain red) $)))$ )

Traffic lights are posted at the entries to an intersection so that some few lanes are controlled by each light. The intersection as a whole can then be classified through the synchronized behavior of the lights. We call an intersection 'safe' if the lights are synchronized so that crossing lanes never show green at the same time.

We introduce a 'conflict point' as an object being related to two or more lanes, that each is controlled by a traffic light. The conflict point is 'resolved' when there is at most one lane whose signal is green at any one time. We obtain the following :

resolved-point $::=$

(and conflict-point

(atmost 1

(occurs

(and lane

(range

$($ when signal green $)))))$

The appearance of the occurs term forces the cycling of green signals to be represented in the development of the resolved point. Only one lane is allowed 
to have a green signal per resolved point object version. Without this occurs term resolved points whose development are unrelated with respect to the green light signaling, are allowed.

A safe intersection is then defined as :

safe-intersection $::=$

(and intersection

(all crossing (always resolved-point)))

We note that (always resolved-point) stresses the fact that every version of the resolved point object is classified as a resolved-point. Without the always the intersection might have been safe only sometimes, which is not our intention.

\section{Discussion}

One of the main arguments for using terminological logics rather than pure first order logic is the computability. Sound and possibly complete algorithms that compute subsumptions is the central issue for providing practical KR services. Until now, as in [Sch90], no algorithms are available for this temporal terminological logic. In this preliminary report, we have concentrated on extending the syntax and semantics of a basic terminological logic to include temporal information about changing extensions of an object and concepts defined in terms of development of objects.

Schmiedel's work [Sch90] bases on Allen's interval calculus [All83] as temporal framework. Concepts are associated with functions from a interval based time domain to sets of individuals. Roles are associated with functions from this interval based time domain to sets of pairs of individuals. (This means that the extension of a concept can change over time.) The use of Allen's interval calculus in defining Schmiedel's standard semantics, implies that if we know that a concept or relation holds over an interval we do not have automatically any information regarding whether the concept or role holds during any sub-interval. This problem can be solved by allowing to associate with a concept or role Shoham's hereditary properties [Sho87]. In our approach we define concepts and roles using pointers, i.e. objects at a specific time where the specificity directly relates to object change. One advantage is that we have exploited LITE's ability to express mixed temporal relations. Therefore roles are allowed to relate objects in different temporal contexts. Further in our approach it is the structure itself which tells us whether we can conclude information about sub-intervals.

The secondary temporal terms have some resemblance with the use of the 
plan description forming operators of Devanbu and Litman [DeL91]. Their system CLASP is used to describe and classify plans. Their 'plan description forming operators' include a SEQUENCE and LOOP operator. For instance

(SEQUENCE $a b c$ )

represents a compound plan consisting of a sequence of a plan of type $a$, a plan of type $b$ and a plan of type $c$. Our seq operator works on roles. A plan could be seen as a transition function between collections (and therefore a role where a pointer in one collection is associated with a pointer in the other collection involving the same object).

$($ seq a b c $)$

would then represent all the instantiations of the compound plan as a transition function.

Recurrence is a crucial element of our common-sense notion of time. As Koomen [Koo91] expresses :

From our earliest days we learn to perceive time as a result of two important cognitive abilities : the awareness of change in the world around us, and the ability to detect regularities in that change. Without change there is no awareness of anything, and without regularities there is awareness of chaos only.

However little work has been done on this topic. In [Koo91] a first order axiomatization based on Allen's interval calculus [All83] is proposed to reason about recurrence. By using the LITE semantics and our plan formation operators, we have made a first step in introducing reasoning about recurrence in terminological logics.

\section{References}

[All83] Allen, J.F., 'Maintaining Knowledge About Temporal Intervals', in Communications of the ACM, Vol 26(11), pp 832-843, 1983.

[Bo*89] Borgida, A., Brachman, R.J., McGuinnes, D.L., Resnick, 'CLASSIC : A Structural Data Model for Objects', in Proceedings of the International Conference on Management of Data - SIGMOD89, pp 59-67, 1989. 
[BrS85] Brachman, R.J., Schmolze, J.G., 'An Overview of the KL-ONE Knowledge Representation System', in Cognitive Science, 9(2), pp 171-216, 1985.

[Br*85] Brachman, R.J., Pigman Gilbert, V., Levesque, H.J., 'An Essential Hybrid Reasoning System : Knowledge and Symbol Level Accounts in KRYPTON', in Proceedings of the International Joint Conference on Artificial Intelligence-IJCAI85, pp 532-539, 1985.

[DeL91] Devanbu, P.T., Litman, D.J., 'Plan-Based Terminological Reasoning', in Proceedings of the Conference on Representation of Knowledge - KR91, pp 128-138, 1991.

[Koo91] Koomen, J.A.G.M., 'Reasoning about Recurrence', in International Journal of Intelligent Systems, Vol 6, pp 461-496, 1991.

[MGB87] MacGregor, R., Bates, R., 'The LOOM Representation Language', Technical Report ISI/RS-87-1988, University of Southern California, Information Science Institute, Marina del Rey, CA, 1987.

[Neb90] Nebel, B., Reasoning and Revision in Hybrid Representation Systems, Lecture Notes in Artificial Intelligence, 422, 1990.

[PaS84] Patel-Schneider, P., 'Small Can Be Beautiful in Knowledge Representation', in Proceedings of the IEEE Workshop on Principles of KnowledgeBased Systems, pp 11-16, 1984.

[Rön90] Rönnquist, R., 'A Logic for Propagation Based Characterisation of Process Behaviour', in Proceedings of the International Symposium on Methodologies for Intelligent Systems - ISMIS90, pp 297-304, 1990.

[Rön92] Rönnquist, R., Theory and Practice of Tense-bound Object References, Ph.D. thesis, nr 270, Dpt. of Computer Science, Linköping University, 1992.

[Sch90] Schmiedel, A., 'A Temporal Terminological Logic', in Proceedings of the National Conference on Artificial Intelligence - AAAI90, pp 640-645, 1990.

[Sho87] Shoham, Y., 'Temporal Logics in AI : Semantical and Ontological Considerations', in Artificial Intelligence, Vol 33(1), pp 89-104, 1987. 